\title{
Modelo Entomológico Determinístico sob Efeito da Pluviosidade para o Aedes aegypti e o Aedes albopictus
}

\author{
Lillia S. Barsante ${ }^{1}$ \\ rizonte, $\mathrm{MG}$ \\ Rodrigo T. N. Cardoso ${ }^{2}$ José L. Acebal ${ }^{3}$ \\ Departamento de Física e Matemática, CEFET-MG, Belo Horizonte, MG \\ Carlos A. Silva ${ }^{4}$ \\ Departamento de Informática, IFMG, Sabará, MG \\ Álvaro E. Eiras ${ }^{5}$ \\ Departamento de Parasitologia, UFMG, Belo Horizonte, MG
}

Programa de Pós Graduação em Modelagem Matemática Computacional, CEFET-MG, Belo Ho-

Resumo. O Aedes aegypti e o Aedes albopictus tem-se destacado na dispersão de diversas arboviroses, dentre elas a dengue, a febre chikungunya e o zika vírus. Recentemente, tornouse crescente pesquisas na área de biomatemática que utilizam modelos entomológicos e/ou epidemiológicos que são capazes de descrever a dinâmica espacial destes vetores, e consequentemente, auxiliar os gestores públicos no controle vetorial e de seu respectivo ciclo de transmissão. Desta forma, realizamos um estudo teórico e numérico, seguido de validação de um sistema entomológico matemático computacional determinístico derivado da literatura, que reproduz a dependência dos parâmetros entomológicos do ciclo de vida do $A$. aegypti e do $A$. albopictus com a pluviosidade acumulada semanalmente de uma determinada área.

Palavras-chave. Aedes aegypti, Aedes albopictus, Modelagem, Pluviosidade

\section{Introdução}

Nos últimos anos tornou-se crescente o número de pessoas infectadas com gravidade variável pelas arboviroses dengue, febre chikungunya e zika vírus. Esforços para criar vacinas e pesquisas que utilizam conceitos de diversas áreas, como biologia, geografia, matemática e computação para descrever, analisar, monitorar e controlar a dinâmica espacial do ciclo de vida dos vetores destas arboviroses tem fomentado o mercado mundial.

No Brasil, o Aedes aegypti e o Aedes albopictus são os principais vetores destas arboviroses apresentando ciclo de vida dividido em duas fases: imatura (ovos, larva e pupas) e alada (mosquito adulto). Estes vetores apresentam grande capacidade de dispersão e

\footnotetext{
${ }^{1}$ lilliabarsante@gmail.com

${ }^{2}$ rodrigoc@des.cefetmg.br

3 acebal@dppg.cefetmg.br

${ }^{4}$ carlos.silva@ifmg.edu.br

5 alvaro@icb.ufmg.br
} 
adaptação ao meio, sendo encontrados com maior frequência em áreas urbanas, suburbanas e rurais pertencentes aos trópicos e sub-trópicos [12]. Segundo [2,4] a probabilidade de dispersão passiva de ovos e larvas em potenciais criadouros destes vetores é maior comparada a dispersão ativa da fase alada. Esta dispersão está relacionada a diversos fatores, como inexistência de controle específico; condições ambientais favoráveis, como o clima e potenciais reservatórios; facilidade dos meios de transporte e saneamento básico precário em algumas localidades [10]. Dentre as variáveis climáticas, iremos destacar a pluviosidade que devido seu acréscimo influencia a quantidade de criadouros potenciais disponíveis para o desenvolvimento da fase imatura destes vetores, além de gerar condições ideais para o desenvolvimento de sua fase alada [7]. Segundo [6] o excesso de chuva poderá eliminar as fases imaturas de potenciais criadouros e desta forma, impactar na mortalidade do vetor. No período de escassez de chuva poderá ocorrer um aumento na população do vetor, pois parte da população humana começa a armazenar água em recipientes abertos que, possivelmente, servirão como potenciais criadouros do vetor [9].

Buscando conferir ao máximo a capacidade preditiva e universalidade, iremos neste trabalho apresentar, analisar e validar um modelo entomológico matemático computacional derivado de [1], em duas cidades brasileiras que contam com dados de pluviosidade acumulada semanalmente juntamente com dados amostrais Índice Médio de Fêmeas Aedes - IMFA, no horizonte de estudo.

\section{Modelagem}

O sistema abordado neste trabalho é uma variação do modelo proposto por [1]. A diferença é caracterizada na parcela não linear que compõe a população de ovos, uma vez que consideramos a probabilidade da fêmeas pós-repasto sanguíneo ovipor em um determinado criadouro dependa da quantidade de toda população da fase imatura do vetor existente neste criadouro e não apenas da população do vetor na fase de ovos. Esta alteração foi embasada em estudos sobre comportamento de oviposição [5]. Desta forma, o sistema de equações diferenciais não lineares que representa a dinâmica das populações do $A$. aegypti e do $A$. albopictus (população de ovos - $E(t)$, população aquática (larvas + pupas) - $A(t)$, população de fêmeas pré-repasto sanguíneo - $F_{1}(t)$, população de fêmeas pós-repasto sanguíneo - $\left.F_{2}(t)\right)$ é descrito por:

$$
\left\{\begin{array}{l}
\dot{E}=\phi(P(t))\left[1-\frac{(E(t)+A(t))}{C(P(t))}\right] F_{2}(t)-\left(\alpha_{1}(P(t))+\mu_{E}(P(t))+\eta_{E}(t)\right) E(t), \\
\dot{A}=\alpha_{1}(P(t)) E(t)-\left(\alpha_{2}(P(t))+\mu_{A}(P(t))+\eta_{A}(t)\right) A(t), \\
\dot{F_{1}}=\alpha_{2}(P(t)) A(t)-\left(\alpha_{3}(P(t))+\mu_{F_{1}}(P(t))+\eta_{F_{1}}(t)\right) F_{1}(t), \\
\dot{F}_{2}=\alpha_{3}(P(t)) F_{1}(t)-\left(\mu_{F_{2}}(P(t))+\eta_{F_{2}}(t)\right) F_{2}(t), \\
\phi, \alpha_{1}, \alpha_{2}, \alpha_{3}, \mu_{E}, \mu_{A}, \mu_{F_{1}}, \mu_{F_{2}}, C, \eta_{E}, \eta_{A}, \eta_{F_{1}}, \eta_{F_{2}}, P \geq 0, \forall t \in \mathbb{R}_{+} .
\end{array}\right.
$$

O parâmetro $\phi$ representa a taxa de oviposição intrínseca por unidade de fêmeas pósrepasto sanguíneo; $C$ representa a capacidade do meio em termos de disponibilidade de alimento, de espaço e outros; $\mu$ e $\eta$ representam as taxas por unidade de indivíduo que morre naturalmente e adicionalmente devido a ação de controle da respectiva população do ciclo destes vetores. As taxas de desenvolvimento são representadas por $\alpha_{1}$ : ovos $\longrightarrow$ 
população aquática; $\alpha_{2}$ : população aquática $\longrightarrow$ fêmeas pré-repasto sanguíneo e $\alpha_{3}$ : prérepasto sanguíneo $\longrightarrow$ fêmeas pós-repasto sanguíneo. Os parâmetros $t$ e $P$ representam, respectivamente, tempo e pluviosidade. A região do sistema (1) com sentido biológico é definida por $\Gamma=\left\{\left(E, A, F_{1}, F_{2}\right): E, A, F_{1}, F_{2} \geq 0\right.$ e $\left.0 \leq E+A \leq C\right\}$, ou seja, todas as populações do sistema são não negativas e a população da fase imatura não excede a capacidade do meio.

Se considerarmos os parâmetros deste sistema invariantes no tempo, pode-se identificar um ponto de equilíbrio trivial $p_{0}$ e o ponto de equilíbrio não trivial $p_{1}$, dados por:

$$
p_{0}=\left(E^{*}, A^{*}, F_{1}^{*}, F_{2}^{*}\right)=(0,0,0,0) \text { e } p_{1}=\left(E^{* *}, A^{* *}, F_{1}^{* *}, F_{2}^{* *}\right)
$$

em que

$$
\begin{gathered}
E^{* *}=\frac{\left(\alpha_{2}+\mu_{A}+\eta_{A}\right)}{\left[\alpha_{1}+\left(\alpha_{2}+\mu_{A}+\eta_{A}\right)\right]} C\left(1-\frac{1}{Q_{0}}\right) ; A^{* *}=\frac{\alpha_{1}}{\left(\alpha_{2}+\mu_{A}+\eta_{A}\right)} E^{* *} \\
F_{1}^{* *}=\frac{\alpha_{2}}{\left(\alpha_{3}+\mu_{F_{1}}+\eta_{F_{1}}\right)} A^{* *} ; F_{2}^{* *}=\frac{\alpha_{3}}{\left(\mu_{F_{2}}+\eta_{F_{2}}\right)} F_{1}^{* *}
\end{gathered}
$$

e o número de descendentes basal $Q_{0}$ é definido por

$$
Q_{0}=\frac{\phi \alpha_{1} \alpha_{2} \alpha_{3}}{\left(\alpha_{1}+\mu_{E}+\eta_{E}\right)\left(\alpha_{2}+\mu_{A}+\eta_{A}\right)\left(\alpha_{3}+\mu_{F_{1}}+\eta_{F_{1}}\right)\left(\mu_{F_{2}}+\eta_{F_{2}}\right)} .
$$

Observe que $Q_{0} \geq 0$, uma vez que todos os parâmetros entomológicos do sistema (1) são reais não negativos. O ponto $p_{1}$ terá significado biológico apenas se $Q_{0} \geq 1$ e será biologicamente trivial se considerarmos $Q_{0}=1$, de modo que $p_{1}=p_{0}$. Utilizando os critérios de Routh-Hurwitz, verifica-se que para $0<Q_{0}<1$, existe um ponto de equilíbrio estável em $p_{0}$ e um ponto de equilíbrio instável em $p_{1}$. A medida que $Q_{0} \longrightarrow 1$, o ponto de equilíbrio instável se aproxima do ponto de equilíbrio estável e $Q_{0}=1$ corresponde a um ponto de bifurcação transcrítica do sistema dinâmico. Finalmente, quando $Q_{0}>1, p_{0}$ se torna instável, e $p_{1}$ estável.

A população do ciclo de vida do A. aegypti e do A. albopictus apresentam uma forte sensibilidade à presença de pouca pluviosidade, produzindo relativamente muitos indivíduos. Contudo, para maiores quantidades de pluviosidade, a resposta da população não guarda a mesma sensibilidade. Este comportamento foi modelado por uma lei de potência, dada por:

$$
\boldsymbol{\pi}=\boldsymbol{\pi}_{\min }+\frac{\left(\boldsymbol{\pi}_{\max }-\boldsymbol{\pi}_{\min }\right)}{\left(P_{1}^{*}-P_{0}\right)^{r_{\pi}}}\left(P-P_{0}\right)^{r_{\pi}},
$$

em que $\pi$ representa genericamente os parâmetros entomológicos $\phi, \alpha_{1}, \alpha_{2}$ e $\alpha_{3}$ do sistema (1); $\pi_{\min }$ e $\pi_{\max }$ representam, respectivamente, o valor mínimo e o valor máximo do parâmetro; a potência $r_{\pi} \in[01]$; $P$ representa a pluviosidade acumulada em cada semana; $P_{0}$ representa a pluviosidade mínima da cidade em estudo; e $P_{1}^{*}$ representa a pluviosidade média semanal de uma região com condições ideais para o desenvolvimento do vetor, que neste caso foi adotada como o valor do limite inferior para classificação de florestas tropicais, (1800 mm/ano). Para os demais parâmetros foi adotada uma dependência linear entre os parâmetros entomológicos do modelo e a pluviosidade acumulada semanalmente ao longo de todo horizonte de estudo. 


\section{Simulação}

O sistema (1) foi resolvido numericamente através do algoritmo de Runge-Kutta de quarta ordem, com passo de 0,01, utilizando o software MATLAB ${ }^{\circledR} 7.12 .0$ (R2011a) em um computador Intel(R) Core(TM) i5 2,5 GHz com 4GB de memória RAM. Por se tratar de uma padronização internacional, a escala temporal deste sistema foi dada em unidades da semana epidemiológica no horizonte de estudo. Adotamos as coordenadas do ponto de equilíbrio não trivial da primeira semana epidemiológica como valor de condição inicial do sistema (1). Os valores máximos e mínimos dos coeficientes entomológicos do sistema (1) são mostrados na Tabela 1 . O parâmetro $\phi_{\min }$ foi obtido de [11]; $\phi_{\max }$ foi obtido de [8]; $\mu_{A}$ foi obtido de [3]; $\alpha_{1}, \alpha_{2}, \mu_{E}, \mu_{F_{1}}$ e $\mu_{F_{2}}$ foram obtidos de [13]; $C$ e $\alpha_{3}$ foram estimados por especialistas da área biológica. Não foram aplicadas ações de controle $\left(\eta_{E}=\eta_{A}=\eta_{F_{1}}=\eta_{F_{2}}=0\right)$ ao longo do horizonte de estudo.

Tabela 1: Faixa de parâmetros adotados no sistema (1).

\begin{tabular}{|c|c|c|c|}
\hline Parâmetro $\left(\right.$ dias $\left.^{-1}\right)$ & Faixa & Parâmetro $\left(\right.$ dias $\left.^{-1}\right)$ & Faixa \\
\hline$\phi$ & $0,5-11,2$ & $\alpha_{1}$ & $0,2-0,5$ \\
\hline$\alpha_{3}$ & $0.5-1$ & $\mu_{F_{1}}=\mu_{F_{2}}$ & $0,0588-0,0286$ \\
\hline$C$ & $1-1$ & $\mu_{E}$ & $0,01-0,01$ \\
\hline$\alpha_{2}$ & $0,0385-0,167$ & $\mu_{A}$ & $0,164-0,132$ \\
\hline
\end{tabular}

A validação do sistema (1) foi realizada de forma qualitativa comparando a evolução da população de fêmeas pós-repasto sanguíneo $F_{2}(t)$ e dados amostrais de IMFA no horizonte referentes às semanas epidemiológicas $23-52$ do ano de 2009 e $1-51$ do ano de 2010 para a cidade de Caratinga (Minas Gerais, Brasil); e 12 - 52 do ano de 2009 e $1-52$ do ano de 2010 para a cidade de Sete Lagoas (Minas Gerais, Brasil), sendo que o valor referente ao dado de monitoramento entomológico da semana epidemiológica 52 do ano de 2009 foi obtido via interpolação linear. Nesta interpolação foram adotados os valores dos dados de monitoramento entomológico das semanas epidemiológicas 51 do ano de 2009 e 1 do ano de 2010. Os dados amostrais de IMFA foram monitorados pelo MIDengue (Ecovec SA) por meio das armadilhas MosquiTRAPs ${ }^{\circledR}$. Este índice corresponde à razão entre o número de fêmeas Aedes em fase de oviposição capturadas pela armadilha MosquiTRAP ${ }^{\circledR}$ e o número total de armadilhas instaladas na região de estudo. O número de armadilhas utilizadas no monitoramento destas cidades foram 162 em Caratinga (Minas Gerais, Brasil) e 497 em Sete Lagoas (Minas Gerais, Brasil). Em ambos os casos, uma armadilha MosquiTRAP ${ }^{\circledR}$ foi instalada no peridomicílio de uma residência respeitando uma distância mínima padrão de 250 metros entre as armadilhas. Os dados amostrais de pluviosidade acumulada semanalmente referente ao período de estudo destas cidades foram obtidos do Instituto Nacional de Pesquisas Espaciais (INPE, Brasil).

$\mathrm{Na}$ validação do sistema (1) foi feita a correlação cruzada entre os dados de IMFA e $F_{2}(t)$, onde definimos uma nova população, $f_{2}(t)$. Buscando coincidir o maior número de 
picos entre as curvas, foi aplicado um fator de escala $\lambda$ em $f_{2}(t)$, dado por

$$
\lambda=\frac{\int_{0}^{N}\left[f_{2}(t)(I(t))^{2}\right] d t}{\int_{0}^{N}\left[I(t)\left(f_{2}(t)\right)^{2}\right] d t},
$$

em que $I(t)$ corresponde aos dados de $I M F A$ e $N$ é o número de semanas epidemiológicas da cidade em estudo. Com intuito de comparar a área entre as curvas foi feito um ajuste pelo método dos mínimos quadrados, resultando o melhor ajuste em $r=0,85$ para $\left(\phi, \alpha_{1}, \alpha_{2}, \alpha_{3}\right)$.

As figuras 1 e 2 ilustram durante o horizonte de estudo que a população de fêmeas pósrepasto sanguíneo acompanha os dados amostrais de IMFA com um atraso médio entre os picos e os vales de duas semanas para a cidade de Caratinga (Minas Gerais, Brasil) e de três semanas para a cidade de Sete Lagoas (Minas Gerais, Brasil). Esta validação pode ser considerada satisfatória, uma vez que abordamos o efeito do tipo logístico da população de ovos sobre a inibição da probabilidade de oviposição em criadouros populosos tanto por ovos quanto por aquática (larvas + pupas) e desconsideramos a influência do controle e de outros fatores climáticos, como temperatura, umidade relativa do ar e altitude na dinâmica do modelo (1). Além disso, devemos ressaltar que os dados amostrais de captura podem sofrer influência de diversas formas, como tecnológicas, humanas e climáticas.
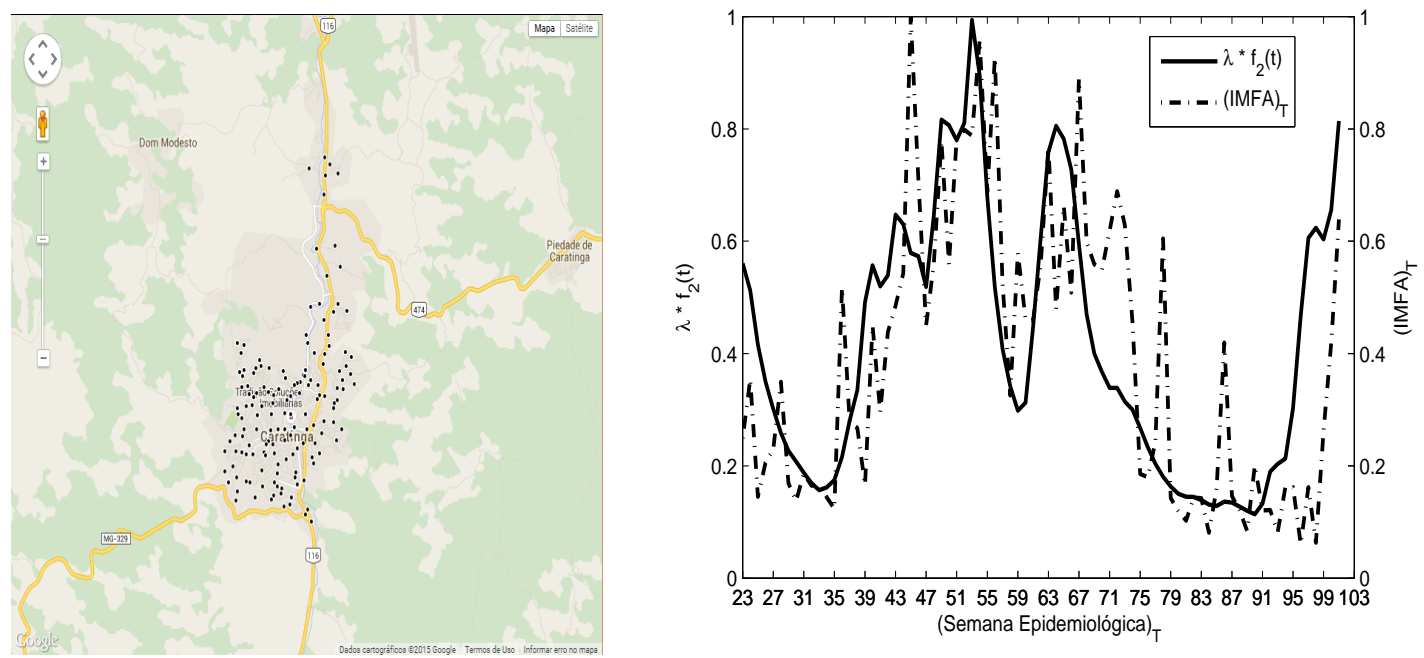

Figura 1: Locais de instalação das armadilhas MosquiTRAPs ${ }^{\circledR}$ e comparação entre a evolução da população de fêmeas pós-repasto sanguíneo e o Índice Médio de Fêmeas Aedes, após correlação e fator de escala, no horizonte de estudo da cidade Caratinga (Minas Gerais, Brasil). 

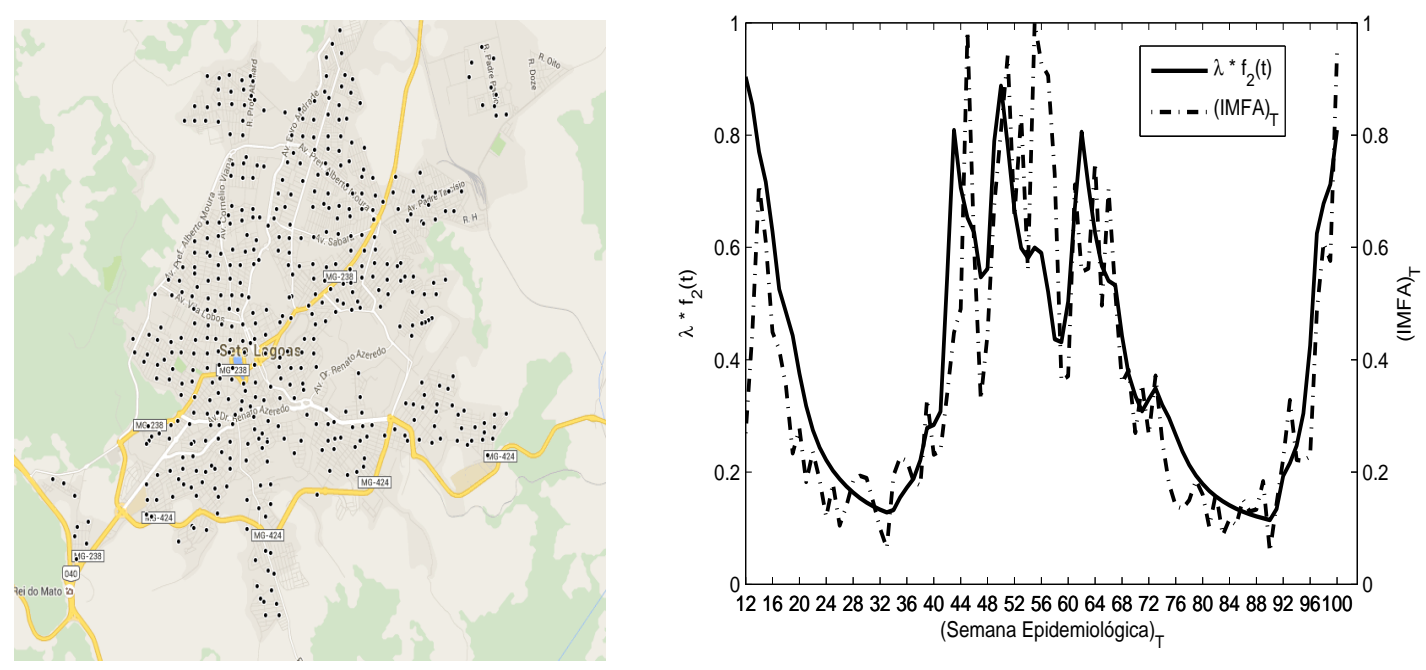

Figura 2: Locais de instalação das armadilhas MosquiTRAPs ${ }^{\circledR}$ e comparação entre a evolução da população de fêmeas pós-repasto sanguíneo e o Índice Médio de Fêmeas Aedes, após correlação e fator de escala, no horizonte de estudo da cidade Sete Lagoas (Minas Gerais, Brasil).

\section{Conclusão}

Diante da necessidade de se aperfeiçoar o conhecimento do ciclo de vida do A. aegypti e do A. albopictus e a fim de promover contribuições às metodologias de controle destes vetores por parte dos gestores públicos, propomos e validamos neste trabalho um sistema dinâmico matemático computacional derivado da literatura. A validação deste sistema pode ser considerada aceitável, principalmente no horizonte chuvoso como pode ser constatado pela proximidade entre a população de fêmeas pós-repasto sanguíneo e dados amostrais de IMFA ao longo do horizonte de estudo. Desta forma, de posse de dados de previsão futura de pluviosidade de uma determinada área, acreditamos que este sistema poderá ser amplamente explorado e desta forma, poderá auxiliar no controle de todo ciclo de vida destes vetores.

\section{Agradecimentos}

Agradecemos ao Centro Federal de Educação Tecnológica de Minas Gerais - CEFETMG e à Coordenação de Aperfeiçoamento de Pessoal de Nível Superior - CAPES pelo incentivo e apoio financeiro para realização deste trabalho.

\section{Referências}

[1] L. S. Barsante, Dependência entre pluviosidade e população de fêmeas Aedes aegypti grávidas descritas através de um sistema dinâmico não linear. Dissertação de Mestrado, CEFET-MG, 2012. 
[2] M. A. R. Costa, A ocorrência do Aedes aegypti na região noroeste do Paraná: um estudo sobre a epidemia da dengue em Paranavaí - 1999, na perspectiva da geografia médica. Dissertação de Mestrado, UNESP, 2001.

[3] C. P. Ferreira e H. M. Yang. Estudo da transmissão da dengue entre os indivíduos em interação com a população de mosquitos Aedes aegypti, TEMA 4(3):323-332, 2003.

[4] Fundação Nacional de Saúde - FUNASA, Dengue: Instruções para pessoal de combate ao vetor. 3, rev. ed. Brasília, 2001.

[5] D. J. Gubler. Studies on the comparative oviposition behavior of Aedes (Stegomyia) albopictus and Aedes (Stegomyia) polynesiensis Marks. Journal of medical entomology, The Oxford University Press, 8(6):675-682, 1971.

[6] C. J. M. Koenraadt and L. C. Harrington. Flushing effect of rain on container inhabiting mosquitoes Aedes aegypti and Culex pipiens (diptera: Culicidae). J. Med. Entomol., 45(1):28-35, 2008.

[7] V. S. G. Neto e J. M. M. Rebêlo. Aspectos epidemiológicos do dengue no município de São Luís, Maranhão, Brasil, 1997-2002. Cad. Saúde Pública, 20(5):1424-1431, 2004.

[8] S. T. R. Pinho, C. P. Ferreira, L. Esteva, F. R. Barreto, V. C. Morato e Silva and M. G. L. Teixeira. Modelling the dynamics of dengue real epidemics, Philosophical Transactions of the Royal Society A: Mathematical, Physical and Engineering Sciences, 368(1933):5679-5693, 2010.

[9] R. J. S. Pontes, J. Freeman, J. W. O. Lima, C. Hodgson and A. Spielman. Vector densities that potentiate dengue outbreaks in a Brazilian city. Am. J. Trop. Med. Hyg., $62(3): 378-383,2000$.

[10] H. H. G. Silva e I. G. Silva. Estudo do ciclo evolutivo do Aedes aegypti (linnaeus,1762) (Diptera: Culicidae) a partir de ovos com quatro meses de estocagem em laboratório. Rev. Pat. Trop., 29(1):95-100, 2000.

[11] R. C. A. Thomé, Controle ótimo aplicado na estratégia de combate ao Aedes aegypti utilizando inseticida e mosquitos estéreis. Tese de Doutorado, UNICAMP, 2007.

[12] M. A. Tolle, Mosquito-borne diseases. Current Problems in Pediatric and Adolescent Health Care, 39(4):97-140, 2009.

[13] H. M. Yang and C. P. Ferreira. Assessing the effects of vector control on dengue transmission. Applied Mathematics and Computation, 198:401-413, 2008. 